\title{
Webquest para fortalecer las competencias digitales en docentes de Bachillerato del circuito 05_06, Distrito 06 de Educación, Guayaquil - 2021
}

\section{Webquest to strengthen digital skills in high school teachers of circuit 05_06, District 06 of Education, Guayaquil - 2021}

DOI: $10.46932 / \mathrm{sfjdv} 3 \mathrm{n} 1-005$

Received in: Dec 30st, 2021

Accepted in: Jan 1th, 2022

\author{
Alvaro Alberto Berruz Guerrero \\ Universidad César Vallejo \\ Maestro en Administración de la Educación \\ Docente de informática y telecomunicaciones \\ Florida norte Cooperativa unidos somos más mz 384 v 4 -Guayaquil-Ecuador \\ E-mail: alvberruz4000@gmail.com. \\ George Artemio Galarza Baque \\ Universidad César Vallejo \\ Maestro en Administración de la Educación \\ Docente de matemática \\ Mapasingue oeste Mz 1380 s. 14 -Guayaquil-Ecuador. \\ E-mail: george_agb@hotmail.com \\ Mario Napoleón Briones Mendoza \\ Universidad César Vallejo \\ Doctor en Educación \\ Docente en filosofía y ciencias sociales \\ $\mathrm{El}$ indio $\mathrm{mz}$ c5 lote 10 Castilla-Piura-Perú \\ E-mail: mbrionesm@ucvvirtual.edu.pe \\ Aurora Victoria Gómez Quintana \\ Universidad César Vallejo \\ Maestra en Administración de la Educación \\ Docente de emprendimiento y gestion \\ La 22 entre el Oro y Vacas Galindo -Guayaquil-Ecuador \\ E-mail: vitocky1@hotmail.com
}

\section{RESUMEN}

La presente investigación tiene como objetivo Determinar la influencia del uso de la Webquest en el fortalecimiento de las competencias digitales en docentes de Bachillerato del circuito 05_06, Distrito 06 de Educación, Guayaquil - 2021. Que contó con la colaboración de docentes de todo el circuito dentro del distrito 6. El tipo de investigación fue cuantitativo, con un diseño cuasi experimental se realizó un pre test y un post test con una población que consto de 80 docentes dividida en 2 grupos de 40 para un grupo experimental y 40 para el grupo de control. La recolección de datos se realizó por medio de una encuesta con preguntas en un cuestionario de tipo Likert. La validez y confiabilidad del instrumento se determinó a través de la revisión de los expertos. Los resultados dentro de la variable competencia digital arrojaron el valor en la aplicación del estadístico "t" de Student, donde se obtuvo un valor p mayor $0,05(, 628)$ y 
una media de 82,35 del grupo experimental y un 84,77 en el grupo control y de la misma forma en la dimensión conocimiento pedagógico se evidencia un aumento del 47,5\% en el nivel avanzado del postest. Concluyendo que la aplicación del Webquest, en donde la mayoría de los resultados se concentraron en los niveles básicos e intermedios posterior a la aplicación del programa elevaron su nivel en todas las dimensiones.

Palabras claves: Webquest, competencias digitales, Conocimiento Tecnológico.

\begin{abstract}
The objective of this research is to determine the influence of the use of the Webquest in the strengthening of digital competences in high school teachers of circuit 05_06, District 06 of Education, Guayaquil 2021. That had the collaboration of teachers from all over the circuit within District 6 . The type of research was quantitative, with a quasi-experimental design, a pre-test and a post-test were carried out. In a population that consisted of 80 teachers divided into 2 groups of 40 for an experimental group and 40 for the control group. Data collection was carried out by means of a survey with questions in a Likert-type questionnaire. The validity and reliability of the instrument was determined through expert review. The results within the digital competence variable yielded the value in the application of Student's " $t$ " statistic, where a p value greater than 0.05 (.628) and a mean of 82.35 of the experimental group and 84,77 in the control group and in the same way in the pedagogical knowledge dimension, there is evidence of an increase of $47.5 \%$ in the advanced level of the post-test. Concluding that the application of Webquest, where most of the results were concentrated in the basic and intermediate levels after the application of the program, raised their level in all dimensions.
\end{abstract}

Keywords: Webquest, digital skills, Technological Knowledge.

\title{
1 INTRODUCCIÓN
}

En la actualidad se están viviendo cambios que vienen de forma muy apresurada, incluso en algunos casos con excesiva celeridad, pero nos parece obvio que por ningún motivo debemos taparnos los ojos e ignorarlo, sino que se requiere de una respuesta reflexiva y consciente (Goig, 2017. p. 3). En este contexto cambiante nos enfrentamos con un sistema escolar que ubica al docente en el centro de la encrucijada, pues son elementos claves en estos procesos de constante cambio; cambio que, además de necesario, se visualiza como algo inaplazable, pues nos encontramos actualmente con formas y enfoques antiguos que no satisfacen carencias de los estudiantes, tampoco de la comunidad, menos a las exigencias del mercado de trabajo.

Con el desarrollo continuo y sostenido de aplicaciones Web se quedan obsoletas dichas aplicaciones rápidamente. En estos tiempos se encuentran diversas plataformas virtuales Web creadas por usuarios, sin embargo, no tienen el soporte adecuado de la ingeniería Web (Preciado, Linaje, Comai y Sanchez-Figueroa, 2005. p. 45). Existen muchas posibilidades y formas de buscar información con las WebQuest y se concibe como una herramienta muy significativa en la práctica laboral de los maestros, pues, con su didáctica los estudiantes desarrollan la capacidad de desempeñarse en otros espacios pedagógicos además del aula, gozando de una variedad de recursos para construir y ejecutar actividades 
de aprendizaje con gran interés (Adell, 2004, p. 34).

Por su lado, Peñaherrera (2015) sostiene que: "Existen elementos críticos en el uso de programas, especialmente en la evaluación del uso de las TIC en programas del sistema educativo ecuatoriano. Estos “nudos" se siguen presentando, la falta de política que relacione el ámbito implementador (institución educativa) y el ámbito diseñador (Gobierno) altera la forma de integración de las TIC” (p.13).

Según Goig (2015) sostiene que: "Una de las herramientas que posibilita desarrollar la competencia digital es la Webquest. Día a día se implementa con mayores recursos esta herramienta. No obstante, la formación del profesorado del profesorado se presenta como una barrera, para implementar este recurso u otros que se pueden utilizar en las sesiones de clase" (p. 2). Por este motivo, en los nuevos planes de estudio de las carreras de educación se implementa el curso "Las TIC implementadas en el ámbito educativo, con el propósito de promover una formación inicial en conocimientos tecnológicos y, utilizándose las TIC como un recurso útil en la pedagogía, desde una visión no sólo instrumental, además, desde una visión didáctica y metodológica.

En esta línea, Romero (2012) considera que: "Es preciso que a los maestros se les acerque a las nuevas tecnologías. Para ello, es necesarios que muestren actitud positiva y abierta ante las nuevas pautas de enseñanza y se vayan despojando de tradicionales formas que vienen aplicando en su trabajo, y empoderarse de estos recursos y nuevas estrategias de enseñanza y sus resultados en los centros que ya las vienen implementando (p. 111).

En el contexto del sistema educativo ecuatoriano, Gonzales, Trelles y Mora (2017) señalan que. "Las herramientas que más implementan los docentes en relación a las TIC son: Word, Exccel, internet y Power Point. Estos recursos son utilizados principalmente por los profesores en su trabajo de aula, un poco menos para el aprendizaje asistido y casi nunca para generar aprendizaje autónomo de los alumnos, no obstante, se requiere iniciar su implementación en la gestión del aprendizaje” (p. 6).

Las TIC en el Plan Nacional de Desarrollo 2015-2020, posibilita la identificación de desafíos a los que se enfrenta el sistema educativo ecuatoriano, así como la necesidad de proponer estrategias que impacten significativamente, estableciendo las condiciones para tener comunidades informadas. Estos son recursos para incorporarse a una sociedad del conocimiento, al promover la sensibilización, difusión e implementación de las TIC, toda vez que, pueden presentarse como una oportunidad de desarrollo, fundamentalmente para las comunidades vulnerables del Ecuador. El distrito 09D06 de Educación en donde se realizó esta investigación, es una institución ubicada en la urbe de Guayaquil que pertenece al Ministerio de Educación y ofrece diferentes servicios a la comunidad educativa e instituciones educativas, las cuales están divididas en diferentes circuitos. De estos circuitos se ha elegido el 05_06, esta cuenta con 62 Instituciones Educativas entre Fiscales, Particulares y Fiscomisionales. 
Analizando el estado de muchas instituciones educativas, se pudo observar que muchos docentes necesitan mejorar sus competencias en el desarrollo de sus clases, aplicando diferentes alternativas tecnológicas que facilitarían su labor y mejorarían el rendimiento académico de sus estudiantes. Este contexto inseguro perjudica los procesos educativos expresada en el desenvolvimiento académico y laboral de los egresados de bachillerato afectando a los miembros de la organización educativa. Ante esta problemática los docentes en los últimos años han tenido que implementar recursos tecnológicos como plataformas virtuales, aplicaciones online, etc., pero muchos de ellos no han podido aprovechar el máximo de estos recursos por el desconocimiento o por que años anteriores no los han necesitado, hoy en día todos los docentes debemos conocer y controlar los recursos digitales y las herramientas que me permiten trabajar con ellos.

En el contexto del sistema educativo ecuatoriano, Gonzales, Trelles y Mora (2017) señalan que. "Las herramientas que más implementan los docentes en relación a las TIC son: Word, Exccel, internet y Power Point. Estos recursos son utilizados principalmente por los profesores en su trabajo de aula, un poco menos para el aprendizaje asistido y casi nunca para generar aprendizaje autónomo de los alumnos, no obstante, se requiere iniciar su implementación en la gestión del aprendizaje” (p. 6).

Analizando el estado de muchas instituciones educativas, se pudo observar que muchos docentes necesitan mejorar sus competencias en el desarrollo de sus clases, aplicando diferentes alternativas tecnológicas que facilitarían su labor y mejorarían el rendimiento académico de sus estudiantes. Este contexto inseguro perjudica los procesos educativos expresada en el desenvolvimiento académico y laboral de los egresados de bachillerato afectando a los miembros de la organización educativa.

En el sentido indicado, la presente investigación se fundamentó en los siguientes antecedentes internacionales: García (2017) en su tesis doctoral: "Competencias digitales en la docencia universitaria del siglo XXI", de la Universidad Complutense de Madrid, tuvo como objetivo de determinar cómo se caracteriza una competencia digital actual, esta Investigación fue de tipos descriptiva, no experimental longitudinal, utilizando como muestra de trabajo a 108 docentes y 135 alumnos La información se recogió utilizando un cuestionario a través del Google Drive, concluyendo que las características que deben tener las competencias digitales son: Hacer uso del internet, búsquedas primordiales, utilización de correo electrónico, Word, aplicaciones multimedia, sistemas operativos, trabajo en línea y uso de herramientas 2.0 .

De la misma manera Lemarié y Gallardo (2017) en su tesis, "Las Webquest como recurso didáctico en el Aprendizaje Basado en Problemas", de la Universidad de Lagos Chile, tuvo como propósito de corroborar su contribución significativa al currículo, orientando el traslado del "saber" hacia el "saber hacer”...Además, si bien las Webquest se convierten en recursos didácticos óptimos, su programación demanda de un periodo adecuado de dedicación del profesor, por lo que se recomienda que se implemente 
principalmente en actividades de mucha importancia para el curso.

Por otro lado tenemos a Burrola (2016) en su tesis doctoral, "Evaluación de las Competencias Básicas en TIC en docentes de México", de la UNED, se propuso un estudio de corte transversal y con enfoque mixto, el cual se desarrolló con muestra de 432 profesores de la Universidad Pública de México,... se concluyó que es importante la implementación de programas que faciliten a las instituciones educativas de recursos tecnológicos, sin embargo, se debe enfatizar que no hay evidencia de que los profesores hagan uso continuo y efectivo de estos recursos en el aula, lo que corroboro el uso básico de estas herramientas.

Gracias a los antecedentes antes mencionados, se puede afirmar que el uso de los tics, siempre ha estado presente en el campo educativo en los diferentes niveles permitiendo a los docentes desarrollar nuevas metodologías y recursos para sus clases y mejorar sus competencias en una época cada vez más digitalizada.

Las versiones actuales de las Webquest, están organizadas en cinco apartados básicos de forma secuencial, son: En primer lugar, la introducción, que describe los propósitos y el ámbito de desarrollo de la Webquest. En segundo lugar, tenemos a la tarea, la cual muestra lo que el estudiante precisa desarrollar en las sesiones, los conocimientos o resultados a alcanzar. En tercer lugar, está el proceso, el cual plantea la distribución y previsión del trabajo y se revelan las herramientas e información que se debe consultar en internet. En cuarto lugar, tenemos a la evaluación, que son los aspectos en el que se consideran ciertos instrumentos que posibilitan la evaluación y comprobación de los aprendido. En quinto lugar, está la conclusión, el cual sintetiza los conocimientos que se han desarrollado y han adquirido los cuales se consideran como significativos. Estos aspectos se asocian con planteamientos de los principios del diseño instructivo de Merrill (2002. p.43), quien considera son fundamentales para el aprendizaje: Problemas, activación, demostración, aplicación e integración.

El método utilizado en las Webquets se compone por el uso de las TIC y de Internet, con planteamientos pedagógicos activos e innovadores, de significativo uso europeo a inicios del siglo $\mathrm{XX}$ (Área, 2006. p. 26). Así mismo, enlaza con la metodología de trabajo por proyectos; con el de los aprendizajes utilizan interrogaciones, indagaciones o problemas y la creación de andamios de Bruner, comprendidos como los apoyos que el docente dispone para que los estudiantes los utilicen y obtengan aprendizajes significativos, en percibir y asignar significados a la información, apropiándoselos e internalizándolos.

También, el Joint Information Systems Committee (JISC) (Hall, 2014) considera elementos fundamentales que forman parte de alfabetización digital: sentirse cómodo en las plataformas virtuales; buscar, evaluar y utilizar información; usar recursos virtuales; comprender el compromiso social, exponer la obtención de resultados, tener conciencia de la identificación analógica, y brindar apoyo en temáticas 
educativas, corporativas y profesionales. No obstante, como señala Eshet-Alkalai (2012), la alfabetización digital va más allá de manejar de forma adecuada los dispositivos digitales; en realidad es la composición de un grupo de capacidades técnico-procesales, cognitivas y socio-emocionales, que se precisan para desenvolverse, educarse y desempeñarse en una comunidad digital (Fraser,Atkins \& Richard, 2013; Nawaz \& Kundi, 2010).

Estas concepciones brindan una definición que sobrepasa el progreso de la capacidad técnica, involucrando aspectos como: administración de la información; asistencia; destreza en la comunicación y para intercambiar información; formulación temas y conocimiento; ética y compromiso; apreciación y solución de problemas; y por último la técnica (Ferrari, 2012). Esta concepción se enmarca en el aspecto tecnológico, informacional y comunicativo, donde el fin transcendental es generar conocimiento. Concepción similar es la propuesta por Esteve y Gisbert (2011) quienes sostienen que la competencia digital viene a ser "las destrezas, conocimientos y actitudes en los campos técnicos, de información, multimedia y comunicativos que tienden el camino a lo que llaman una alfabetización diversa y compleja" (p.55).

Por su lado, Larraz (2013) comprende a la competencia digital como: "las acciones de alfabetización para actuar de forma efectiva, demostrando buena conducta ciudadana en relación a la identidad digital" (p. 14). Además, Durán (2019), sostiene que la competencia digital es: "El grupo de conocimientos, habilidades y actitudes que se precisan para utilizar de manera efectiva las TIC desde sus diversas vertientes y en diferentes contextos, llevándolos así a la mejora de una alfabetización digital múltiple" (p. 24).

En la actualidad, al profesor se le visualiza como el principal agente para orientar los procesos de perfeccionamiento de la competencia digital en los estudiantes del presente siglo (Llorente, 2008; Salinas y Silva, 2014). Para ello se precisa contar con profesores que demuestren habilidades digitales, con destrezas, actitudes y conocimientos tecnológicos, generando un contexto de aprendizaje fortalecido con la implementación de las tecnologías digitales (Suárez, Almerich, Gargallo y Aliaga, 2010). Así, los recursos digitales deberían ser utilizados para la optimización e innovación de trabajo pedagógico, progreso profesional y su identidad docente (Esteve, Gisbert y Lázaro, 2016; Fraser, Atkins y Hall, 2013; Silva, Miranda, Gisbert, Morales y Onetto, 2016); creando contextos enseñanza y aprendizaje, direccionados por herramientas digitales, a través del uso de métodos específicos, desde una visión donde el discente es el protagonista del aprendizaje (Silva et al.; 2016).

La función del profesor ha logrado desarrollarse de forma muy compleja, tanto así que no es suficiente con el dominio de su especialidad, nos ha avisado Darling-Hammond (2001), su responsabilidad va más allá de la comunicación de un conocimiento específico, que, a través de las TIC, implica niveles altos de estudio y dedicación para implementar el progreso de la habilidad creativa, de colaboración y de 
invención en el proceso de enseñanza y aprendizaje. Diversos autores (Esteve, 2015; Mineduc-Enlaces 2008 y 2011) nos proponen la urgencia de formar profesores con capacidades digitales para una conveniente inclusión de las TIC en las prácticas pedagógicas (Silva, 2012; Salinas y Silva, 2014).

Parra concretar una pertinente concepción del conjunto de competencias digitales de los profesores, Gutiérrez (2011) sostiene que se debe iniciar de indicadores que se utilicen como referentes tanto para tener en cuenta profesores competentes en este sentido, como para la formulación de programas de formación en habilidades digitales (p. 19). En esta línea, Prendes y Gutiérrez (2012), sostienen que los patrones sobre competencias TIC de los profesores nos dan la posibilidad de obtener una guía que permite apreciar a aquellos profesores que han desarrollado habilidades digitales.

De los modelos que analizan las competencias digitales docentes, se tiene el Modelo de Competencia Digital Docente actualizado (Prendes, 2017), el cual comprende 5 aspectos de la competencia digital:

Primero tenemos, la Técnica: Implica la utilización de las diversas herramientas que se derivan de las TIC y la solución de dificultades técnicas. A continuación, la Informacional/comunicativa: Se considera en este componente la indagación y administración de la información, cómo el profesor aplica criterios de criticidad a la información y la comunicación en línea. Después, la Educativa: Este aspecto se compone de el esbozo, administración y valoración de contextos enriquecidos/virtuales, la inclusión efectiva de las TIC en los procedimientos pedagógicos, así como la interacción, asesoramiento, seguimiento y valoración de los estudiantes. También, la Analítica: La dimensión considera el estudio y reflexión particular del profesor en relación a la utilización de las herramientas virtuales, así como la gestión del progreso profesional y la formación continua. A continuación, tenemos la Socio-ética: Se considera en este aspecto la conciencia de la influencia social de las tecnologías, el liderazgo y administración de la innovación con tecnologías virtuales, la reserva y seguridad en la web, la salud en el ámbito laboral y la ética en la utilización responsable de las TIC.

Primero tenemos al Conocimiento disciplinar. Se refiere a los contenidos y a las destrezas de la materia que se desarrollarán en el proceso pedagógico, y por tanto, difiere en cada área de contenido, y comprende la elección de los métodos y las estrategias didácticas pertinentes para desarrollar conocimientos en los contenidos tratados, en segundo, tenemos al Conocimiento tecnológico. Hace referencia al modo en que haciendo uso de los recursos tecnológicos, se innovan formas de desarrollar, exponer y simbolizar el contenido de diversas disciplinas y por ultimo tenemos el Contenido pedagógico. Considera la forma en como las diversas herramientas tecnológicas se utilizan en el proceso pedagógico, y asimismo, comprende cómo a utilización de las tecnologías influye en los estilos de enseñanza de los docentes. 
Según el modelo TPACK (Koehler \& Mishra, 2008; Mishra, 2011), solamente a partir de una adecuada combinación de conocimiento tecnológico, disciplinar y didáctico-pedagógico podremos hacer servir todas las potencialidades de las TIC para facilitar los procesos de enseñanza aprendizaje. Como plantea Salinas (2014) este modelo TPACK aporta las dimensiones adecuadas para el estudio y la comprensión de esta competencia digital docente, ya que no se puede entender el conocimiento tecnológico desligado de la metodología adecuada a los nuevos escenarios de aprendizaje.

\section{METODOLOGÍA}

La investigación se sostuvo en el enfoque cuantitativo, por tanto, fue de dirección positivista, pues, como indica Hernández, Fernández y Baptista (2014) estos estudios están implícitas en el contexto de las ciencias empírico-analíticas, por tanto, pretenden comprobar hipótesis con la finalidad alcanzar a generalizar los resultados. Según La Torre, Del Rincón y Arnal, (2003) el tipo de la investigación es experimental, en las cuales se maneja una variable (independiente) para estimar los cambios en otra (dependiente). Se buscó estimar en qué medida la aplicación de una Webquest fortalece las competencias digitales de los docentes de bachillerato del circuito 05_06, Distrito 06 de Educación, Guayaquil - 2021.

El diseño de la investigación a utilizó es el cuasi experimental con preprueba y posprueba, un grupo experimental y un grupo de control (Petrosko, 2004. Citado en Hernández et al 2014. p. 145)

La población se conformó por los sujetos u objetos que personifican a las unidades de análisis, demostrando rasgos habituales (Hernández et al. 2014). En este caso la población estará conformada por los 400 docentes del circuito 05_06, Distrito 06 de Educación, Guayaquil.

La muestra fue considerada como una parte de la población sobre quien se compilo datos, además, de forma previa se precisó y delimito, resultando un subgrupo característico de la población (Hernández et al. p.173). La muestra en este caso fue determinada por conveniencia del investigador, quien fijó los dos grupos de trabajo, uno experimental y otro de control, con 40 docentes en cada uno de ellos.

\section{RESULTADOS}

\subsection{RESULTADOS DESCRIPTIVOS}

\subsubsection{Objetivo Especifico 01}

OE1: Establecer el nivel de competencias digitales en docentes de bachillerato del grupo experimental y de control, del circuito 05_06, Distrito 06 de Educación, Guayaquil - 2021, antes de aplicar la Webquest. 
Tabla 1 Nivel de competencia digital en docentes de bachillerato del grupo experimental y grupo de control

\begin{tabular}{|c|c|c|c|c|}
\hline \multirow{2}{*}{ Nivel } & \multicolumn{2}{|c|}{ G. Experimental } & \multicolumn{2}{|c|}{ G. Control } \\
\hline & $\mathrm{N}^{0}$ & $\%$ & $\mathrm{~N}^{0}$ & $\%$ \\
\hline Básico & 13 & $32,5 \%$ & 10 & $25,0 \%$ \\
\hline Intermedio & 24 & $60,0 \%$ & 28 & $70,0 \%$ \\
\hline Avanzado & 3 & $7,5 \%$ & 2 & $5,0 \%$ \\
\hline Total & 40 & $100,0 \%$ & 40 & $100,0 \%$ \\
\hline
\end{tabular}

Fuente: Encuesta aplicada a docentes de bachillerato del circuito 05_06 del distrito 06 de educación

En la tabla 1 se observa el nivel de desarrollo de competencia digitales, en docentes del grupo experimental con un 32,5\% en el nivel Básico, mientras que por el grupo de control se observa un $25 \%$ en el mismo nivel. Asimismo, se tiene $60 \%$ de los encuestados del grupo experimental en el nivel intermedio y un 70\% del grupo de control en el mismo nivel. Asimismo, se aprecia en el grupo experimental un 7,5\% que se ubican en el nivel avanzado y $5 \%$ del grupo control. Estos datos permiten deducir que ambos grupos tienen valores similares, sin diferencias significativas.

\subsection{CONTRASTACIÓN DE HIPÓTESIS}

Tabla 2 Prueba de Normalidad de Competencia Digitales

\begin{tabular}{lccc|ccc}
\hline & \multicolumn{3}{c|}{ Kolmogorov-Smirnov } & \multicolumn{3}{c}{ Shapiro-Wilk } \\
\cline { 2 - 7 } & Estadístico & Gl & Sig. & Estadístico & gl & Sig. \\
\hline Competencia Digital_Exp &, 066 & 40 &, $200^{*}$ &, 988 & 40 &, 935 \\
Competencia Digital_Ctrl &, 094 & 40 &, $200^{*}$ &, 981 & 40 &, 738 \\
\hline
\end{tabular}

Fuente: Encuesta aplicada al grupo experimental y grupo de control

Los resultados de la tabla 2, se puede observar que existe un alto grado de significatividad de ,935 del grupo experimental y ,738 del grupo de control, ambos valores superan el valor mínimo de ,005 además se debe considerar que la población es menor a 50, por lo tanto, se considera los resultados de Shapiro-Wilk. por los tanto se aplicó el estadístico T student.

\subsubsection{Comprobación de las Hipótesis 01}

$\mathrm{H}_{\mathrm{i}} 1$. Existen entre los docentes de bachillerato del grupo experimental y de control, del circuito 05_06, Distrito 06 de Educación, Guayaquil - 2021, diferencias significativas antes de la aplicación de la Webquest. 
Tabla 3 Prueba de $\mathrm{T}$ de muestras independientes

\begin{tabular}{|c|c|c|c|c|c|c|c|}
\hline & Competencia Digitales & $\mathrm{N}$ & Media & $\begin{array}{c}\text { Desv. } \\
\text { Desviación }\end{array}$ & $t$ & gl & $\begin{array}{c}\text { Sig. } \\
\text { (bilateral) }\end{array}$ \\
\hline \multirow{2}{*}{ Competencia Digitales } & Grupo Experimental & 40 & 82,3500 & 22,61330 &,- 486 & 78 &, 628 \\
\hline & Grupo de Control & 40 & 84,7750 & 21,96674 &,- 486 & 77,934 & 628 \\
\hline
\end{tabular}

Fuente: Datos de la encuesta al grupo experimental y el grupo de control

En base a los resultados de la tabla 3 según la prueba aplicada T - Student se puede apreciar que entre los resultados de la "Media" no hay diferencias significativas entre el grupo experimental con 82,35 y el grupo de control con 84,77 sobre el nivel de competencia digital; esto también se respalda con la significatividad bilateral de ,628 en ambos grupos el cual es superior a ,005 lo que significa que no hay diferencias significativas entre los grupos de estudio, siendo éstos homogéneos. En conclusión, el grupo experimental presento características similares en el nivel de competencias digitales con el grupo de control antes de implementar el programa, por lo tanto, la hipótesis queda probada.

\subsubsection{Objetivo Especifico 02}

OE2: Identificar diferencias del nivel de competencia digital en las dimensiones conocimiento disciplinar, conocimiento tecnológico y conocimiento pedagógico, en docentes de bachillerato del grupo experimental y grupo de control circuito 05_06, Distrito 06 de Educación, Guayaquil - 2021, antes de aplicar de la Webquest.

Tabla 4 Tabla personalizada del nivel de competencia digital por dimensiones del grupo experimental y grupo de control

\begin{tabular}{lcccccccc}
\hline & \multicolumn{2}{c}{ Básico } & \multicolumn{2}{c}{ Intermedio } & \multicolumn{2}{c}{ Avanzado } & \multicolumn{2}{c}{ Total } \\
\cline { 2 - 10 } & $\mathrm{Fi}$ & $\%$ & $\mathrm{Fi}$ & $\%$ & $\mathrm{Fi}$ & $\%$ & $\mathrm{Fi}$ & $\%$ \\
\hline Conocimiento Disciplinar GE & 19 & $47,5 \%$ & 16 & $40,0 \%$ & 5 & $12,5 \%$ & 40 & $100,0 \%$ \\
Conocimiento Disciplinar GC & 15 & $37,5 \%$ & 22 & $55,0 \%$ & 3 & $7,5 \%$ & 40 & $100,0 \%$ \\
Conocimiento Tecnológico GE & 5 & $12,5 \%$ & 29 & $72,5 \%$ & 6 & $15,0 \%$ & 40 & $100,0 \%$ \\
Conocimiento Tecnológico GC & 5 & $12,5 \%$ & 27 & $67,5 \%$ & 8 & $20,0 \%$ & 40 & $100,0 \%$ \\
Conocimiento Pedagógico GE & 4 & $10,0 \%$ & 29 & $72,5 \%$ & 7 & $17,5 \%$ & 40 & $100,0 \%$ \\
Conocimiento Pedagógico GC & 4 & $10,0 \%$ & 32 & $80,0 \%$ & 4 & $10,0 \%$ & 40 & $100,0 \%$ \\
\hline
\end{tabular}

Fuente: Encuesta aplicada a docentes de bachillerato del circuito 05_06 del distrito 06 de educación

En la tabla 4 se observa el nivel de desarrollo de la dimensión conocimiento disciplinar, en docentes del grupo experimental con un $47,5 \%$ en el nivel Básico, mientras que por el grupo de control se observa un $37,5 \%$ en el mismo nivel. Asimismo, se tiene $40 \%$ de los encuestados del grupo experimental en el nivel intermedio y un $55 \%$ del grupo de control en el mismo nivel. Asimismo, se 
aprecia en el grupo experimental un $12.5 \%$ que se ubican en el nivel avanzado y $7.5 \%$ del grupo control. Estos datos permiten deducir que ambos grupos tienen valores similares, sin diferencias significativas.

De la misma forma, se observa el nivel de desarrollo de la dimensión conocimiento tecnológico, en docentes del grupo experimental con un $72,5 \%$ en el nivel Intermedio, mientras que por el grupo de control se observa un 67,5\% en el mismo nivel. Asimismo, se tiene $15 \%$ de los encuestados del grupo experimental en el nivel Avanzado y un 20\% del grupo de control en el mismo nivel. Asimismo, en el nivel básico en ambos grupos es del 12,5\%. Se puede concluir que los grupos tienen valores homogéneos, sin diferencias significativas.

Del mismo modo, en el nivel de desarrollo de la dimensión conocimiento pedagógico, en docentes del grupo experimental con un $72,5 \%$ en el nivel Intermedio, mientras que por el grupo de control se observa un $80 \%$ en el mismo nivel. Asimismo, se tiene 17,5\% de los encuestados del grupo experimental y $10 \%$ del grupo de control en el nivel Avanzado. Asimismo, en el nivel básico en ambos grupos es del $10 \%$. Se puedo concluir que los grupos tienen valores parecidos, sin diferencias significativas.

\subsubsection{Comprobación de las Hipótesis 02}

$\mathrm{H}_{\mathrm{i}}$ 2: Existen diferencias significativas en nivel de competencia digital en las dimensiones conocimiento disciplinar, conocimiento tecnológico y conocimiento pedagógico en docentes de bachillerato del grupo experimental y grupo de control circuito 05_06, Distrito 06 de Educación, Guayaquil - 2021, antes de aplicar de la Webquest.

Tabla 5 Prueba de T de muestras independientes

\begin{tabular}{|c|c|c|c|c|c|c|c|}
\hline Dimensión & Grupos & $\mathrm{N}$ & Media & $\begin{array}{c}\text { Desv. } \\
\text { Desviación }\end{array}$ & $\mathrm{t}$ & gl & $\begin{array}{c}\text { Sig. } \\
\text { (bilateral) }\end{array}$ \\
\hline \multirow{2}{*}{ Conocimiento Disciplinar } & G. Experimental & 40 & 34,0000 & 14,29542 &,- 527 & 78 &, 600 \\
\hline & G. Control & 40 & 35,6250 & 13,27169 &,- 527 & 77,573 &, 600 \\
\hline \multirow{2}{*}{ Conocimiento tecnológico } & G. Experimental & 40 & 23,8750 & 5,82298 &,- 382 & 78 &, 703 \\
\hline & G. Control & 40 & 24,3750 & 5,87340 &,- 382 & 77,994 &, 703 \\
\hline \multirow{2}{*}{ Conocimiento pedagógico } & G. Experimental & 40 & 24,6750 & 5,13104 &, 305 & 78 &, 761 \\
\hline & G. Control & 40 & 24,3250 & 5,13603 & ,305 & 78,000 &, 761 \\
\hline
\end{tabular}

Fuente: Datos de la encuesta al grupo experimental y el grupo de control

En base a los resultados de la tabla 5 según la prueba aplicada $\mathrm{T}$ - Student se puede apreciar que entre los resultados de la "Media" no hay diferencias significativas entre el grupo experimental con 34 y 
el grupo de control con 35,62 sobre el nivel de conocimiento disciplinar; esto también se respalda con la significatividad bilateral de ,600 en ambos grupos el cual es superior a ,005 lo que significa que no hay diferencias significativas entre los grupos de estudio, siendo éstos homogéneos. En conclusión, el grupo experimental presento características similares en el nivel de conocimiento disciplinar con el grupo de control antes de implementar el programa.

De la misma manera, se puede apreciar que entre los resultados de la "Media" no hay diferencias significativas entre el grupo experimental con 23,87 y el grupo de control con 24,37 sobre el nivel de conocimiento tecnológico; esto también se respalda con la significatividad bilateral de ,703 en ambos grupos el cual es superior a ,005 lo que significa que no hay diferencias significativas entre los grupos de estudio, siendo éstos similares. En conclusión, el grupo experimental presento características equivalentes en el nivel de conocimiento tecnológico con el grupo de control antes de implementar el programa.

También, se puede observar que entre los resultados de la "Media" no hubo diferencias significativas entre el grupo experimental con 24,67 y el grupo de control con 24,32 sobre el nivel de conocimiento pedagógico; esto también se respalda con la significatividad bilateral de ,761 en ambos grupos el cual es superior a ,005 lo que significa que no hay diferencias significativas entre los grupos de estudio, siendo éstos similares. En conclusión, el grupo experimental presento características equivalentes en el nivel de conocimiento pedagógico con el grupo de control antes de implementar el programa.

\subsubsection{Objetivo Especifico 03}

OE3: Establecer el nivel de competencias digitales en docentes de bachillerato del grupo experimental, del circuito 05_06, Distrito 06 de Educación, Guayaquil - 2021, antes y después de aplicar la Webquest.

Tabla 6 Nivel de competencia digital en docentes del grupo experimental antes y después del programa

\begin{tabular}{ccccc}
\hline & \multicolumn{4}{c}{ G. Experimental } \\
\hline \multirow{2}{*}{ Nivel } & \multicolumn{2}{c}{ Pretest } & \multicolumn{2}{c}{ Postest } \\
\cline { 2 - 5 } & $\mathrm{N}^{\circ}$ & $\%$ & $\mathrm{~N}^{\circ}$ & $\%$ \\
\hline Básico & 13 & $32,5 \%$ & 0 & $0,0 \%$ \\
Intermedio & 24 & $60,0 \%$ & 22 & $55,0 \%$ \\
Avanzado & 3 & $7,5 \%$ & 18 & $45,0 \%$ \\
Total & 40 & $100,0 \%$ & 40 & $100,0 \%$ \\
\hline
\end{tabular}

Fuente: Encuesta aplicada a docentes de bachillerato del circuito 05_06 del distrito 06 de educación

De acuerdo con la tabla 6 se puede apreciar que, al comprar los resultados obtenidos por el grupo experimental, respecto al nivel de competencias digitales, en el pretest el 32,5\% de docentes muestran un nivel básico en esta competencia, mientras que, en el postest, se muestra una reducción total (0\%) en este nivel. Así mismo, en el pretest, hubo un $60 \%$ de docentes en un nivel intermedio, lo cual, en el post test 
disminuye al 55\% en este nivel. También se puedo apreciar que en el pretest del grupo experimental el 7,5\% de docentes se encuentra en un nivel avanzado, mientras que en el postest se alcanzó un 45\% de los docentes en este nivel, ya que la diferencia de los niveles anteriores (32,5\% del Nivel básico y 5\% del nivel intermedio) pasaron a ubicarse al nivel avanzado. Esto quiere decir, que en el postest existe un incremento significativo en el nivel de las competencias digitales

\subsubsection{Comprobación de las Hipótesis 03}

$\mathrm{H}_{\mathrm{i}} 3$ : La aplicación de la Webquest aumenta significativamente las competencias digitales de los docentes de bachillerato del grupo experimental del circuito 05_06, Distrito 06 de Educación, Guayaquil -2021 ,

Tabla 7 Prueba de T de significatividad de la Webquest

\begin{tabular}{cccccccc}
\hline & Test & $\mathrm{N}$ & Media & Desv. Desviación & $\mathrm{t}$ & $\mathrm{gl}$ & Sig. (bilateral) \\
\hline \multirow{3}{*}{ Competencias digitales } & Pretest & 40 & 82,3500 & 22,61330 & $-7,353$ & 78 &, 000 \\
& Postest & 40 & 115,3750 & 17,19226 & $-7,353$ & 72,794 &, 000 \\
\hline
\end{tabular}

Fuente: Encuesta aplicada a docentes de bachillerato del circuito 05_06 del distrito 06 de educación

La tabla 7, se observa que al aplicar la prueba " $t$ " de Student a los datos obtenidos en el pretest y postest del grupo experimental, se encuentran una diferencia de medias entre los dos momentos de 33,03 con un valor " $t$ " de $-7,353 \mathrm{y}$ el Sig. (bilateral) de, 000 donde $<0,05$ lo que significa que existen diferencias significativas entre el pretest y postest, habiendo aumentado el nivel de competencia digitales en el postest, por lo tanto, se da por aceptada la hipótesis.

\subsubsection{Objetivo General}

O.G.: Determinar la influencia del uso de la Webquest en el fortalecimiento de las competencias digitales en docentes de bachillerato del circuito 05_06, Distrito 06 de Educación, Guayaquil - 2021

Tabla 8 Nivel de competencia digital en docentes del grupo experimental antes y después del programa por dimensiones

\begin{tabular}{ccccccccc}
\hline \multirow{2}{*}{ Dimensión } & \multicolumn{2}{c}{ BÁSICO } & INTERMEDIO & AVANZADO & \multicolumn{2}{c}{ Total } \\
\cline { 2 - 10 } & $\mathrm{fi}$ & $\%$ & $\mathrm{fi}$ & $\%$ & $\mathrm{fi}$ & $\%$ & $\mathrm{fi}$ & $\%$ \\
\hline Conocimiento Disciplinar PRETEST & 19 & $47,5 \%$ & 16 & $40,0 \%$ & 5 & $12,5 \%$ & 40 & $100,0 \%$ \\
Conocimiento Disciplinar POSTEST & 0 & $0,0 \%$ & 27 & $67,5 \%$ & 13 & $32,5 \%$ & 40 & $100,0 \%$ \\
\hline Conocimiento tecnológico PRETEST & 5 & $12,5 \%$ & 29 & $72,5 \%$ & 6 & $15,0 \%$ & 40 & $100,0 \%$ \\
Conocimiento tecnológico POSTEST & 0 & $0,0 \%$ & 13 & $32,5 \%$ & 27 & $67,5 \%$ & 40 & $100,0 \%$ \\
\hline Conocimiento pedagógico PRETEST & 4 & $10,0 \%$ & 29 & $72,5 \%$ & 7 & $17,5 \%$ & 40 & $100,0 \%$ \\
Conocimiento pedagógico POSTEST & 0 & $0,0 \%$ & 14 & $35,0 \%$ & 26 & $65,0 \%$ & 40 & $100,0 \%$ \\
\hline
\end{tabular}

Fuente: Encuesta aplicada a docentes de bachillerato del circuito 05_06 del distrito 06 de educación 
En la tabla 8 se observa los datos antes y después de la aplicación de la Webquest de la dimensión, Conocimiento Disciplinar, que, en la mayoría de los docentes, antes de aplicar la Webquest se ubicaba en un nivel básico del $47,5 \%$, un nivel intermedio del $40 \%$ y un $12 \%$ en el nivel avanzado, de los cuales después aplicar la Webquest se concentraron e incrementaron el nivel intermedio con un 67,5\% y un 32,5\% en el nivel avanzado. En lo que corresponde a la dimensión, Conocimiento Tecnológico, antes de aplicar la Webquest, en el nivel básico del pretest tiene un 12,5\%, un 72,5\% en el nivel intermedio y un $15 \%$ en el nivel avanzado, de los cuales después de aplicar la Webquest se evidencia mejoras en el nivel intermedio con un 32,5\% y un $67,5 \%$ en el nivel avanzado. De la misma manera la dimensión, Conocimiento Pedagógico, antes de la aplicación de la Webquest, el 10\% de los docentes se encuentran en un nivel básico, también se puede observar un 72,5\% en el nivel intermedio y un 17,5\% en el nivel avanzado, de los cuales después de aplicar la Webquest existe una disminución en el nivel intermedio del $35 \%$, pero un incremento del $65 \%$ en el nivel avanzado. Esto quiere decir que la aplicación de la Webquest, aumento los niveles de la competencia digital en cada una de sus dimensiones en los docentes del grupo experimental, donde se llevó la investigación.

\subsection{HIPÓTESIS GENERAL}

La aplicación de la Webquest tiene un efecto positivo y significativo en las competencias digitales de los docentes de bachillerato del circuito 05_06, Distrito 06 de Educación, Guayaquil - 2021

Tabla 9 Prueba de T de significatividad de la Webquest - diferencias emparejadas

\begin{tabular}{|c|c|c|c|c|c|c|c|c|}
\hline Dimensiones & Test & $\mathrm{N}$ & Media & $\begin{array}{c}\text { Diferencia } \\
\text { Media }\end{array}$ & $\begin{array}{c}\text { Desv. } \\
\text { Desviación }\end{array}$ & $t$ & gl & $\begin{array}{c}\text { Sig. } \\
\text { (bilateral) }\end{array}$ \\
\hline Conocimiento & Pretest & 40 & 34,0000 & \multirow{2}{*}{18,58} & 14,29542 & $-6,809$ & 78 &, 000 \\
\hline Disciplinar & Postest & 40 & 52,5750 & & 9,66062 & $-6,809$ & 68,474 &, 000 \\
\hline \multirow{2}{*}{$\begin{array}{l}\text { Conocimiento } \\
\text { tecnológico }\end{array}$} & Pretest & 40 & 23,8750 & \multirow{2}{*}{7,60} & 5,82298 & $-6,342$ & 78 &, 000 \\
\hline & Postest & 40 & 31,4750 & & 4,85158 & $-6,342$ & 75,539 &, 000 \\
\hline \multirow{2}{*}{$\begin{array}{l}\text { Conocimiento } \\
\text { pedagógico }\end{array}$} & Pretest & 40 & 24,6750 & \multirow{2}{*}{6,65} & 5,13104 & $-6,039$ & 78 &, 000 \\
\hline & Postest & 40 & 31,3250 & & 4,70890 & $-6,039$ & 77,432 &, 000 \\
\hline
\end{tabular}

Fuente: Encuesta aplicada a docentes de bachillerato del circuito 05_06 del distrito 06 de educación

En la tabla 9, al someter los datos a la prueba " $t$ " de Student, se observa en cada una de las dimensiones de la variable competencia digitales, que existen diferencias de "medias" significativas, de 18,58 en la dimensión, Conocimiento Disciplinar, 7,60 en la dimensión, Conocimiento tecnológico y 6,65 en la dimensión, Conocimiento Pedagógico, por lo tanto, de acuerdo con la tabla 8 y los resultados de la presente tabla, se puede afirmar que la aplicación de la Webquest aumento los niveles de competencias digitales de los docentes de bachillerato, lo que lleva a aceptar la hipótesis general de la investigación. 


\section{DISCUSIÓN}

En esta investigación a través de sus objetivos se pudo determinar la influencia del uso de la Webquest en el fortalecimiento de las competencias digitales en docentes de Bachillerato del circuito 05_06, Distrito 06 de Educación, Guayaquil - 2021, donde se demuestra una mejora en el nivel de competencia digital de los docentes.

En el objetivo específico 1 después de proceder a establecer el nivel de competencias digitales en docentes de bachillerato del grupo experimental y de control, del circuito 05_06, Distrito 06 de Educación, Guayaquil - 2021, antes de aplicar la Webquest, la mayoría concuerdan en el nivel medio, donde está ubicado el $60 \%$ del grupo experimental y 70\% de grupo control. Esto se confirmó con la aplicación del estadístico " $t$ " de Student, donde se obtuvo un valor p mayor $0,05(, 628)$ y una media de 82,35 del grupo experimental y un 84,77 en el grupo de control, lo que indica que antes de aplicar la Webquest, la mayoría de docentes, no existe diferencias significativas. Resultados similares se encontró al valorar cada una de las dimensiones de los niveles de competencia digital: En el nivel literal, tanto el grupo experimental como el de control se ubican en el nivel básico o intermedio, esto aplica también en el nivel inferencial y critical. Estos resultados son contrarios con la investigación de Jiménez (2017) titulada "Evaluación de la eficacia del programa de educación de Gestión empresarial”, en donde los docentes utilizaron pocas estrategias de innovación con TIC, ya que la conducta no fue observada en un 35\% y poco observada en un 30\%, lo cual centra los más altos porcentajes. A su vez, coinciden con la teoría Gutiérrez (2011) sostiene que se debe iniciar de indicadores que se utilicen como referentes tanto para tener en cuenta profesores competentes en este sentido, como para la formulación de programas de formación en habilidades digitales.

En el objetivo específico 2 después de proceder a identificar las diferencias del nivel de competencia digital en las dimensiones conocimiento disciplinar, conocimiento tecnológico y conocimiento pedagógico, en docentes de bachillerato del grupo experimental y grupo de control circuito 05_06, Distrito 06 de Educación, Guayaquil - 2021, antes de aplicar de la Webquest, en donde la mayoría de los resultados se concentran en los niveles básicos e intermedios en la dimensión Conocimiento Disciplinar del GE se encuentra en el nivel Básico con 47,5\% y en el nivel intermedio del 55\% del GC, Asimismo, en la dimensión Conocimiento Tecnológico, cuenta un nivel intermedio del 72,5\% del GE y del 67,5\% en el GC en el mismo nivel. También en la dimensión Conocimiento Pedagógico, con el 72,5\% del nivel intermedio del GE, mientras que el GC tiene un $80 \%$ en el mismo nivel del GC. Esto se confirmó con la aplicación del estadístico " $t$ ” de Student, a cada una de las dimensiones, donde se obtuvo un valor p mayor 0,05, en la dimensión disciplinar (,600), en la dimensión tecnológica $(, 703)$ y en a dimensión pedagogía (,761), lo que indica que antes de aplicar la Webquest, la mayoría de docentes, no existe diferencias significativas. Estos resultados concuerdan con los de Burrola (2016) titulada "Evaluación de las Competencias Básicas en TIC en docentes de México” en los que los resultados en lo que se refiere al 
factor de frecuencia con que utiliza el computador, para preparar e impartir clases, se observa que un $42.8 \%$ de la población menciono emplearlo más de 5 veces seguida en una semana, además por un $26.8 \%$ de 2-3 veces a la semana; del factor que utiliza procesadores de texto tenemos un $45.2 \%$ de los maestros que lo utilizan más de 5 veces a la semana seguido y un 23\% que lo utiliza de 2-3 veces por semana. El factor que utiliza hoja de cálculo muestra que el 35\% de la muestra en docentes la utilizan de 0-1 vez por semana seguido por un $29 \%$ que la utiliza de 2-3 veces por semana; en lo que respecta a adopción en rangos el 71.53\%. A su vez, coindice con la postura teórica de Keating y Evans (2001) en la que define al conocimiento disciplinar como los contenidos y a las destrezas de la materia que se desarrollarán en el proceso pedagógico, y por tanto, difiere en cada área de contenido, y comprende la elección de los métodos y las estrategias didácticas pertinentes para desarrollar conocimientos en los contenidos tratados.

En el objetivo específico 3 después de proceder a establecer el nivel de competencias digitales en docentes de bachillerato del grupo experimental, del circuito 05_06, Distrito 06 de Educación, Guayaquil - 2021, antes y después de aplicar la Webquest, muestra en el postest que la mayoría se encuentran en los niveles medio con un 55\% y avanzado con un $45 \%$ a diferencia del pretest, que contaba con un nivel básico del 32,5\%, un nivel intermedio del 60\%. Esto se confirmó con la aplicación del estadístico "t" de Student, donde se obtuvo un valor p menor a $0,05(, 000)$ y una media de 82,35 en el pretest del grupo experimental y un 115,37 del postest en el mismo grupo, lo que indica que después de aplicar la Webquest, la mayoría de docentes mejoraron, demostrando unas diferencias significativas a beneficios de los docentes después del postest. En el nivel literal, el grupo experimental se ubican en los niveles intermedio y avanzado, esto aplica también en el nivel inferencial y critical. Estos resultados coinciden con la investigación de Esteve (2016) titulada "La competencia digital docente" en los mismo se encontraron que la dimensión aprendizaje y creatividad muestra un $47 \%$ con un nivel medio alto en su capacidad y un $46 \%$ en el nivel medio de su capacidad y finalmente un $7 \%$ se percibe poco capaz. A su vez, coincide con la postura teórica de Zhao (2003) en la que define al conocimiento tecnológico como el modo en que haciendo uso de los recursos tecnológicos, se innovan formas de desarrollar, exponer y simbolizar el contenido de diversas disciplinas.

El objetivo general después de proceder a determinar la influencia del uso de la Webquest en el fortalecimiento de las competencias digitales en docentes de bachillerato del circuito 05_06, Distrito 06 de Educación, Guayaquil - 2021, muestra un aumento en la dimensión de conocimiento disciplinar del $27,5 \%$ en el nivel intermedio y un $20 \%$ en el nivel avanzado del postest, los mismos valores que unidos conformaban el nivel básico del pretes y con un 47,5\% del grupo experimental. Así mismo, tenemos un aumento en la dimensión conocimiento tecnológico del 52,5\% en el nivel avanzado del postest los cuales se encontraban en el nivel básico con un $12,5 \%$ y nivel intermedio con un $40 \%$ en el pretest del grupo antes mencionado. También en la dimensión conocimiento pedagógico se evidencia un aumento del 
47,5\% en el nivel avanzado del postest, los cuales se los puede identificar entre el nivel básico del 10\% y 37,5\% del nivel intermedio del pretest. Esto se confirmó con la aplicación del estadístico "t" de Student, donde se obtuvo un valor p menor a $0,05(, 000)$ y una diferencia de media de 18,58 de la dimensión disciplinar del grupo experimental, un 7,60 en la dimensión tecnológico y un 6,65 en la dimensión pedagógica en el mismo grupo, lo que indica que después de aplicar la Webquest, la mayoría de docentes mejoraron, demostrando unas diferencias significativas a beneficios de los docentes después del postest. En el nivel literal, el grupo experimental se ubican en los niveles intermedio y avanzado, esto aplica también en el nivel inferencial y critical. Estos resultados coinciden con la investigación de Vargas (2018) titulada "La competencia digital y el uso de aplicaciones web 2.0 en docentes" en los que existe una correlación positiva moderada entre sus variables con un $\mathrm{r}=, 891$ y en aspectos como utilización de herramientas tipo office se encontró que el $32 \%$ está de altamente satisfecho y el uso del trabajo colaborativo el $88 \%$ está de acuerdo con su uso. A su vez, la postura teórica de (Schmidt et al., 2009) señala que el contenido pedagógico es la forma en como las diversas herramientas tecnológicas se utilizan en el proceso pedagógico, y asimismo, comprende cómo a utilización de las tecnologías influye en los estilos de enseñanza de los docentes.

\section{CONCLUSIÓN}

Con respecto al objetivo general, la aplicación del Webquest influyó significativamente en el fortalecimiento de las competencias digitales, y según la tabla 9 se evidencia una diferencia significativa en los grupos lo que indica la incidencia del Webquest en los docentes de bachillerato para sus dimensiones disciplinar, tecnológico y pedagógico, mejorando de esta forma la labor propia del docente dentro de su ámbito laboral.

La aplicación del Webquest influyó significativamente en la dimensión conocimiento disciplinar según la tabla 9, donde se evidencia una diferencia significativa en los grupos lo que indica la incidencia del Webquest mejoro los aspectos del conocimiento enfocados en la disciplina y orden de los docentes.

La aplicación del Webquest influyó significativamente en la dimensión conocimiento tecnológico según la tabla 9, donde se evidencia una diferencia significativa en los grupos lo que indica la incidencia del Webquest mejoro los aspectos tecnológicos lo cual conlleva a un mejor uso de las TIC y un mejor desenvolvimiento frente a las tecnologías.

La aplicación del Webquest influyó significativamente en la dimensión conocimiento pedagógico según la tabla 9, en el cual se evidencia una diferencia significativa en los grupos lo que indica la incidencia del Webquest mejoro los aspectos relacionados al curriculum del maestro, dado que mostraron mejor orden y mejoraron sus competencias frente a los procesos de enseñanza y aprendizaje. 


\section{REFERENCIAS}

Adell, J. (2004). Internet en el aula: Las WebQuest. Edutec. Revista Electrónica de Tecnología Educativa, 17. Recuperado 4 octubre 2014, de http://edutec.rediris.es/Revelec2/revelec17/adell_16a.htm

Ackermann, E. (2001) Piaget's Constructivism, Papert's Constructionism: What's the difference? Recuperado de: 〈http://learning.media.mit.edu/content/ publications/EA.Piaget_Papert.pdf >.

Area, M. (2006) Hablemos más de métodos de enseñanza y menos de máquinas digitales: los proyectos de trabajo a través de la WWW». Cooperación Educativa, núm. 79, p. 26-32, 2005-2006.

Burrola, M. (2016) Evaluación de las Competencias Básicas en TIC en docentes de educación superior en México (tesis doctoral) Universidad de educación a distancia UNED

Comisión Europea (2006) Competencias clave para el aprendizaje permanente. Recomendación 2006/962/CE del Parlamento Europeo y del Consejo, de 18 de diciembre de 2006, sobre las competencias clave para el aprendizaje permanente [Diario Oficial L 394 de 30.12.2006]

Darling Hammond, L. (2001). El derecho de aprender: crear buenas escuelas para todos. Ariel.

Dodge, B. (2001) Rubric for Evaluating WebQuests. San Diego: San Diego State University, 2001b.

Dodge, B. (2000) Thinking Visually with WebQuests. San Diego: San Diego State University, 2000 Recuperado de hhttp://edweb.sdsu.edu/Webquest/tv/\#>.

Dodge, B. (1995). Origen del Concepto Webquest. Comunitat Catalana de Webquest, 1995 Recuperado de <http://www.xtec.net/ cbarba1/Articles/concepteWQ.htm>.

Eshet-Alalai, Y. (2012). Thinking in the digital era: A revised model for digital literacy. Issues in Informing Science and Information Technology, 9, 257-276.

Esteve, F., Gisbert, M. y Lázaro, J. (2016). La competencia digital de los futuros docen-tes: ¿cómo se ven los actuales estudiantes de educación? Perspectiva Educacional, $55 \quad$ (2), 3452. DOI:10.4151/07189729Vol.55Iss.2Art.412

Esteve, F. (2015). La competencia digital del futuro docente: análisis de su auto percep-ción y evaluación de su desempeño por medio de un entorno 3D (Tesis Doctoral). Universitat Rovira i Virgili, Tarragona

Esteve, F. y Gisbert, M. (2011). El nuevo paradigma de aprendizaje y nuevas tecnologías. REDU. Revista De Docencia Universitaria, 9(3), 55-73.

Ferrari, A. (2012). Digital competence in practice: An analysis of frameworks. Sevilla: European Commission, Joint Research Centre (JRC).

Fraser, J., Atkins, L., y Richard, H. (2013). DigiLit leicester. Supporting teachers, pro-moting digital literacy, transforming learning. Leicester City Council.

Fraser, J., Atkins, L., \& Richard, H. (2013). DigiLit leicester. Supporting teachers, promoting digital literacy, transforming learning. Leicester City Council.

García, F (2017) Competencias digitales en la docencia universitaria del siglo XXI. (tesis doctoral ) Universidad computlense de Madrid. 
Goig, R. (2017) La webquest como recurso para adquirir la Competencia digital en la etapa de Educación infantil. La necesidad de Formación del profesorado en las tic. Universidad Nacional de Educación a Distancia España.

González, V. (2007) Del profesor tradicional al diseñador educativo apoyado en la Web. Enseñar y aprender con WebQuest. Gobierno del Principado de Asturias, Consejería de Educación y Ciencia, 2007

Gonzales, M. Trelles, C y Mora, J. (2017) Manejo Docente de las Tecnologías de la Información y Comunicación. Cuenca, Ecuador (artículo científico) Universidad de Cuenca.

Gutiérrez, I. (2011). Competencias del profesorado universitario en relación al uso de tecnologías de la información y comunicación: Análisis de la situación en España y propuesta de un modelo de formación.

Hernández, S., Fernández, A. Baptista, A. (2014). Metodología de la Investigación. México. Editorial Mc Graw Hill

Keating, T., \& Evans, E. (2001). Three computers in the back of the classroom:

Pre-service teachers' conceptions of technology integration. InR. Carlsen, N. Davis, J. Price, R. Weber, \& D. Willis (Eds.), Society for Information Technology and Teacher Education Annual, 2001 (pp. 1671- 1676). Norfolk, VA: Association for the Advancement of Computing in Education.

Koehler, M. J. (2008). Handbook of technological pedagogicalcontent knowledge (TPCK) for educators. New York: Routledge

Koehler, M. J., \& Mishra, P. (2008). Handbook of technological pedagogical content knowledge (TPCK) for educators. New York: Routledge

Larraz, V. (2013). La competència digital a la Universitat. (Tesis Doctoral. Programa de doctorat de la Universitat d'Andorra.) Recuperado de: http://hdl.handle.net/10803/113431

La Torre, Del Rincón y Arnal, (2003) Métodos de investigación. Editores línea México

Lemarié, F. y Gallardo, A. (2017) Las Webquest como recurso didáctico en el Aprendizaje Basado en Problemas, (tesis doctoral) Universidad de Lagos Chile.

Llorente, M.C. (2008). Aspectos fundamentales de la formación del profesorado en TIC. PixelBit: Revista de medios y educación, 31, 121130

Martin, A. (2005). DigEuLit--a european framework for digital literacy: A progress report. Journal of eLiteracy, 2(2), 130-136

Merrill. M. (2002) Firts principles onf Instruction». Educational Technology, Research and Development, vol. 50, núm, 3, p. 43-59 (Versión en línea en: 〈http://id2.usu.edu/Papers/5FirstPrinciples.PDF>

Mertler, C. (2001) Designing scoring rubrics for your classroom». Practical Assessment, Research \& Evaluation, vol. 7, núm. 25

Meyers, E. M., Erickson, I., \& Small, R. V. (2013). Digital literacy and informal learning environments: An introduction. Learning, Media and Technology, 38(4), 355-367. doi:10.1080/17439884.2013.783597

MineducEnlaces (2011). Actualización de Competencias y Estándares TIC en la Profe-sión docente. Ministerio de Educación, Chile

Mineduc-Enlaces (2008). Estándares TIC para la Formación Inicial Docente: Una pro-puesta en el contexto Chileno. Ministerio de Educación, Chile 
Mishra, P. (2011). The seven trans-disciplinary habits of mind: Extending the TPACK framework towards 21st century learning. Educational Technology, (March-April).

Nawaz, A., \& Kundi, G. M. (2010). Digital literacy: An analysis of the contemporary paradigms. Journal of Science and Technology Education Research, 1(2), 19-29.

Peñaherrera, M. (2012). Uso de Tic en Escuelas Públicas de Ecuador: Análisis, Reflexiones y Valoraciones. EDUTEC. Revista Electrónica de Tecnología Educativa, 40, 1-16. Retrieved from http://www.edutec.es/revista/index.php/edutec-e/article/view/364

Preciado, J.C., Linaje, M., Sánchez, F. y Comai, S. (2005). Necessity of methodologies to model Rich Internet Applications. 7th IEEE International Symposium on Web Site Evolution, Budapest, Hungary, IEEE.

Prendes, M. y Gutiérrez, I. (2012). Competencia para el uso de TIC de los futuros maestros. Revista Comunicar, 35, 175-182. doi: 10.3916/C35-2010-03-11.

Quintana, A. Higueras, E. (2007) Les Webquests, una metodología d'aprenentatge cooperatiu, basada en la cerca guiada d'informació per la Xarxa. Romero, M. (2012) Las webquests: una herramienta para introducir las tecnologías de la información y la comunicación en el aula. Conservatorio Superior de Música de Málaga (España).

Salinas, J. (2004). Innovación docente y uso de las TIC en la enseñanza universitaria. Revista de Universidad y Sociedad del Conocimiento, RUSC, 1(1).

Salinas, J. y Silva J. (2014). Innovación con TIC en la formación inicial docente en Ibe-roamérica. En Silva. J. y Salinas, J. (Coords) Innovación con TIC en Formación Inicial Docentes: Aspectos teóricos y casos concretos. (pp 1233). Santiago.

Schmidt, D. A., Baran, E., Thompson, A. D., Mishra, P., Koehler, M. J., \& Shin, T. S. (2009). Technological pedagogical content knowledge (TPACK): The development and validation of an assessment instrument for preservice teachers. Journal of Research on Computing in Education, 42(2), 123-149.

Silva J, Gisbert M., Morales MJ, Onetto A. (2016a). Evaluación de la competencia digital en la formación inicial docente: Una propuesta en el contexto Chileno - Urugua-yo. En R. Roig Vila (ed.)

Silva, J. (2012). Estándares TIC para la Formación Inicial Docente. Una política en el contexto chileno. Education Policy Analysis Archives, 20 (7), 136. DOI:10.14507/epaa.v20n7.2012

Starr, L. (2000) The Frank Lloyd Wright of Learning Environments! Education World. Recuperado de $<$ http://www.educationworld.com/a_issues/chat/chat015.shtml > (Versión en español en: $<$ http://www.eduteka.org/Entrevista11.php> [Consulta: 28 de octubre de 2007]).

Suárez, J, Almerich, G, Gargallo, B y Aliaga, F (2010). Las competencias en TIC del profesorado y su relación con el uso de los recursos tecnológicos, Archivos Analí-ticos de Políticas Educativas, 18 (10).

Zhao, Y. (2003). What teachers should know about technology: Perspectives and practices. Greenwich, CT: Information Age Publishing 